\title{
Intervención cognitivo-conductual para el control metabólico de la diabetes mellitus tipo 2: un estudio piloto
}

\section{Cognitive-behavioral intervention for the metabolic control of type 2 diabetes mellitus: a pilot study}

\author{
Joel Omar González-Cantero \\ Universidad de Guadalajara, México \\ Roberto Oropeza Tena \\ Universidad Michoacana de San Nicolás de Hidalgo, México \\ Jorge Armando Casillas Rodríguez \\ Universidad Tecnológica de Guadalajara, México
}

Rec (10 de Junio de 2018) Acept (08 de Julio de 2019)

\begin{abstract}
Resumen
En México, una de cada cuatro personas con diabetes mellitus tipo 2 (DM2) tiene un control aceptable de la enfermedad. Consecuentemente, el propósito de este estudio fue determinar el cambio clínico de una intervención cognitivo-conductual en el control metabólico (CM) de la DM2 y variables psicológicas asociadas (malestar emocional asociado a diabetes mellitus [MEADM], sintomatología depresiva y bienestar psicológico). Se utilizó un diseño cuasiexperimental pretest-postest con seguimiento a los tres meses. Los resultados muestran un efecto muy alto de la intervención en el MEADM del pretest al postest $(g=1.399)$ y al seguimiento $(g=1.478)$; un efecto moderado del pretest al postest $(g=0.461)$ y alto del pretest al postest ( $g=1.036)$ en el bienestar psicológico. Se necesita más tiempo para que la HbA1c y la sintomatología depresiva lleguen a rangos de control.

Palabras clave: diabetes mellitus tipo 2, control metabólico, estrés, bienestar psicológico, intervención cognitivo-conductual.
\end{abstract}

\begin{abstract}
In Mexico, one of four people with type 2 diabetes mellitus has an acceptable control. Thus, the purpose of this study was to determine the clinical change of a cognitive-behavioral intervention on metabolic control and associated psychological variables (emotional distress, depressive symptomatology and psychological well-being) in people with this disease. A quasi-experimental pretest posttest design with a follow-up of one and three months was used. Results show a very high effect on the emotional distress to the pretest to posttest $(\mathrm{g}=1.399)$ and to the follow-up $(\mathrm{g}=1.478)$; a moderate effect from pretest to posttest $(\mathrm{g}=0.461)$ and high from pretest to posttest $(g=1.036)$ in psychological well-being. More time is needed for the HbA1c and depressive symptomatology reach ranges of control.

Key words: Type 2 diabetes mellitus; metabolic control, stress, psychological well-being, cognitive-behavioral intervention.
\end{abstract}

Correspondencia: Joel González joel.gonzalez@academicos.udg.mx; Roberto Oropeza dipumsnh@yahoo.com; Jorge Casillas jorge. casillasr@uteg.edu.mx

Agradecimientos: Al Programa para el Desarrollo Profesional Docente, Tipo Superior en la modalidad Apoyo a Incorporación de Nuevo Profesor de Tiempo Completo (No. UDG-PTC-1423) 


\section{Introducción}

La Federación Internacional de Diabetes (2015) señala que en México entre el 40 y $60 \%$ de las muertes en personas menores de 60 años son a causa de la diabetes y que el gasto promedio por persona al año en dólares está en un rango de 500 a 2000. El problema se agrava porque se estima que solo el $25 \%$ de las personas que padecen la enfermedad, tienen un adecuado CM (Flores, Reyes, Villalpando, Reynoso \& Hernández, 2012).

Diversas intervenciones, la mayoría de ellas cognitivo-conductuales, para el control de la DM2 han mostrado resultados eficaces; no obstante, como señalan González-Cantero \& Oropeza (2016), han tenido poco control metodológico (particularmente, cuentan con poco control de factores de validación interna).

Es importante que las intervenciones psicológicas aborden variables motivacionales que ayuden a las personas a controlar su enfermedad (García \& Morales, 2015). Así, entre las variables asociadas al CM de la DM2 destaca el bajo nivel de estrés (Ortiz, Baeza-Rivera \& Myers, 2013; Ortiz, Ortiz, Gatica \& Gómez, 2011; Torres \& Piña, 2010); la sintomatología depresiva (Robles, 2002) y el bienestar psicológico (Lucha-López et al., 2017).

Por lo anterior, el propósito del presente trabajo fue determinar el cambio clínico de una intervención cognitivo-conductual (INCOCOM) para el CM y variables psicológicas asociadas (MEAD, sintomatología depresiva y bienestar psicológico) en pacientes con DM2.

\section{Método}

\section{Diseño}

El diseño fue cuasiexperimental de dos grupos independientes (grupo experimental de intervención psicológica individual protocolizada $[\mathrm{GE}]$ y grupo control $[\mathrm{GC}]$ ) con medidas repetidas (pretest, postest y seguimiento a tres meses).

\section{Participantes}

La muestra se formó por nueve personas, ocho mujeres y un hombre. El GE se integró por cuatro personas y el GC por cinco. La media de edad fue de $60.33(D E=8.01)$ y de años de escolaridad fue de $8.22(D E=3.59)$. Ocho eran casados y uno soltero. El tiempo de padecimiento tuvo un promedio de 10.56 años $(D E=4.41)$. De los nueve participantes, tres reportaron complicaciones de la DM2.

\section{Instrumentos}

Cuestionario de variables sociodemográficas y de la enfermedad. Con base en este cuestionario se recolectó información sobre los indicadores metabólicos y antropométricos para el CM de la DM2 de acuerdo con lo que estipula la NOM-015-SSA2-2010, Para la prevención, tratamiento y control de la diabetes mellitus (HbA1c $<7 \%$; colesterol total $<200 \mathrm{mg} / \mathrm{dL}$; colesterol HDL $>40$ hombres, $>50$ mujeres; triglicéridos $<150 \mathrm{mg} / \mathrm{dL}$; circunferencia abdominal $<80 \mathrm{~cm}$ mujeres, $<90 \mathrm{~cm}$ hombres). Además se agregaron la circunferencia de cintura y de cadera.

Cuestionario de Malestar Emocional Asociado a Diabetes. Fue creado por Polonsky et al. (1995), y adaptado y validado por Del Castillo \& Reyes-Lagunes (2010) para población mexicana en una muestra de 240 pacientes con DM2, quienes reportan un alfa de Cronbach general de .90. Esta escala tipo Likert consta de 16 reactivos con cinco opciones de respuesta.

Escala de Autoevaluación para la Depresión de Zung. Creada por Zung (1965) y validada en población clínica en México por Rivera, Corrales \& Piña (2007), quienes reportan un alfa de Cronbach global de 0.88. Se conforma por 20 reactivos con cuatro opciones de respuesta.

Escala de Bienestar Psicológico de Ryff. Fue elaborada por Ryff (1989) y validada en México por MedinaCalvillo, Gutiérrez-Hernández \& Padrós-Blázquez (2013) cuyo alfa de Cronbach fue de .91. Es una escala tipo Likert de 39 ítems con 6 opciones de respuesta. 


\section{Procedimiento}

Para aumentar la fiabilidad de la aplicación de la INCOCOM por el terapeuta, se elaboró un manual y se entrenó a un terapeuta (con grado de maestría y experiencia en psicoterapia cognitivo-conductual) durante 20 horas. Además, se videograbaron todas las sesiones de un paciente para evaluar la adherencia al manual por parte del psicoterapeuta.

La INCOCOM se conforma de 12 sesiones con duración de una hora y media cada una. Los tópicos de las sesiones fueron los siguientes: Sesión 1, Encuadre y aspectos básicos de la DM2; Sesión 2, Alimentación saludable I para el control de la DM2; Sesión 3, Alimentación saludable II para el control de la DM2; Sesión 4, Actividad física para el control de la DM2; Sesión 5; Habilidades asertivas para el rechazo de alimentos no saludables; Sesión 6, Manejo de pensamientos perjudiciales para el control de la DM2; Sesión 7, Relajación para mejorar el control metabólico; Sesión 8, Autocontrol para el control de la DM2; Sesión 9, Solución de problemas para el control de la DM2; Sesión 10 Cierre y evaluación; Sesión 11, Seguimiento al primer mes; Sesión 12, Seguimiento a los 3 meses.

\section{Análisis de datos}

Inicialmente se realizó un análisis de homogeneidad a través de la X2 de Fisher con base en las variables sociodemograficas y de la enfermedad. Se usó la prueba de Wilcoxon para identificar las diferencias de los GC y GE, del pretest al postest y del pretest al seguimiento en los indicadores del CM de la DM2 y las variables psicológicas asociadas al mismo. Enseguida se utilizó la $g$ de Hedges para evaluar el tamaño del efecto, relacionado con cambios clínicamente significativos. Finalmente, con la U de Mann-Whitney se buscaron diferencias entre el GC y GE en los indicadores del CM de la DM2 y las variables psicológicas asociadas en dos momentos, en el pretest y el postest.

\section{Aspectos éticos}

El presente estudio, catalogado como de riesgo mínimo, se sujetó a los lineamientos establecidos en el Código Ético del Psicólogo que emite la Sociedad Mexicana de Psicología y el Consejo Nacional para la Enseñanza e Investigación en Psicología (Sociedad Mexicana de Psicología, 2010).

\section{Resultados}

Con base en el análisis de la homogeneidad a través de la $X^{2}$ de Fisher se identificó homogeneidad entre el GC y GE por sexo $\left(\chi^{2}=.900, p=.343\right)$; estado civil $\left(\chi^{2}=1.406, p=.236\right)$; complicaciones $\left(\chi^{2}=3.600, p=.058\right)$ y comorbilidad $\left(\chi^{2}=.032, p=.858\right)$. La única variable en donde se identificaron diferencias al inicio fue en la variable tiempo de padecimiento $(Z=1.50, p=.032)$ como se observa en la Tabla 1. 
Tabla 1. Medias (M), desviaciones estándar (DE) y diferencias iniciales entre el GC y el GE

\begin{tabular}{lccc}
\hline & $\begin{array}{c}\text { Grupo control } \\
(\mathrm{n}=5)\end{array}$ & $\begin{array}{c}\text { Grupo experimental } \\
(\mathrm{n}=4)\end{array}$ & $Z(p)$ \\
\hline Variable & $M(D E)$ & $M(D E)$ & $8.00(.618)$ \\
Edad & $62.80(7.53)$ & $57.25(8.53)$ & $1.50(.032)$ \\
Tiempo de padecimiento & $13.00(4.35)$ & $7.50(2.08)$ & $3.00(.086)$ \\
HbA1c & $6.34(.59)$ & $8.43(2.42)$ & $10.00(1.00)$ \\
Colesterol total & $196.18(34.31)$ & $184.37(15.79)$ & $9.00(.806)$ \\
Colesterol HDL & $43.97(6.37)$ & $47.97(13.46)$ & $5.00(.221)$ \\
Triglicéridos & $169.19(64.25)$ & $127.47(72.21)$ & $8.50(.730)$ \\
Peso & $70.68(14.98)$ & $70.95(8.61)$ & $8.00(.730)$ \\
Circunferencia cintura & $98.80(9.90)$ & $97.32(14.69)$ & $9.50(.905)$ \\
Circunferencia cadera & $106.00(5.52)$ & $108.87(11.87)$ & $10.00(1.00)$ \\
MEADM & $10.60(6.42)$ & $51.75(6.89)$ & $8.50(.712)$ \\
Sintomatología depresiva & $42.60(4.50)$ & $45.00(13.68)$ & $6.00(.327)$ \\
Bienestar psicológico & $181.60(17.81)$ & $147.25(11.58)$ & \\
\hline
\end{tabular}

Fuente: elaboración propia

En los resultados del pretest al postest en el GE destaca el efecto mediano $(g=.504)$ en el colesterol total, pero en sentido desfavorable, es decir aumentó, aunque se mantuvo dentro del rango de control $(200 \mathrm{mg} / \mathrm{dL})$ como se observa en la Tabla 2.

Tabla 2. Comparaciones y tamaño del efecto del CM de la DM2 del GC y del GE del pretest al postest

\begin{tabular}{|c|c|c|c|c|c|c|c|c|}
\hline & \multicolumn{4}{|c|}{ Grupo control $(n=5)$} & \multicolumn{4}{|c|}{ Grupo experimental $(n=4)$} \\
\hline Variable & $\begin{array}{c}\text { Pretest } \\
M \\
(D E)\end{array}$ & $\begin{array}{c}\text { Postest } \\
M \\
(D E)\end{array}$ & $\begin{array}{c}Z \\
(p)\end{array}$ & $g$ & $\begin{array}{c}\text { Pretest } \\
M \\
(D E)\end{array}$ & $\begin{array}{c}\text { Postest } \\
M \\
(D E)\end{array}$ & $\begin{array}{c}Z \\
(p)\end{array}$ & $g$ \\
\hline $\mathrm{HbA} 1 \mathrm{c}$ & $\begin{array}{l}6.34 \\
(.59)\end{array}$ & $\begin{array}{l}6.29 \\
(.86)\end{array}$ & $\begin{array}{l}-.135 \\
(.893)\end{array}$ & .061 & $\begin{array}{c}8.43 \\
(2.42)\end{array}$ & $\begin{array}{c}8.14 \\
(1.70)\end{array}$ & $\begin{array}{l}-.730 \\
(.465)\end{array}$ & .120 \\
\hline Colesterol total & $\begin{array}{l}196.18 \\
(34.31)\end{array}$ & $\begin{array}{l}202.98 \\
(11.55)\end{array}$ & $\begin{array}{l}-.405 \\
(.686)\end{array}$ & .239 & $\begin{array}{l}184.37 \\
(15.79)\end{array}$ & $\begin{array}{l}194.07 \\
(17.61)\end{array}$ & $\begin{array}{l}-.365 \\
(.715)\end{array}$ & .504 \\
\hline Colesterol HDL & $\begin{array}{l}43.97 \\
(6.37)\end{array}$ & $\begin{array}{l}40.16 \\
(9.58)\end{array}$ & $\begin{array}{l}-.944 \\
(.345)\end{array}$ & .423 & $\begin{array}{l}47.97 \\
(13.46)\end{array}$ & $\begin{array}{c}52.92 \\
(10.86)\end{array}$ & $\begin{array}{l}-.730 \\
(.465)\end{array}$ & .351 \\
\hline Triglicéridos & $\begin{array}{l}169.19 \\
(64.25)\end{array}$ & $\begin{array}{l}158.60 \\
(80.3)\end{array}$ & $\begin{array}{l}-.405 \\
(.686)\end{array}$ & .131 & $\begin{array}{l}127.47 \\
(72.21)\end{array}$ & $\begin{array}{l}117.32 \\
(36.44)\end{array}$ & $\begin{array}{l}-.365 \\
(.715)\end{array}$ & .154 \\
\hline Peso & $\begin{array}{c}70.68 \\
(14.98)\end{array}$ & $\begin{array}{c}69.78 \\
(14.25)\end{array}$ & $\begin{array}{l}-.816 \\
(.414)\end{array}$ & .055 & $\begin{array}{l}70.95 \\
(8.61)\end{array}$ & $\begin{array}{l}70.40 \\
(8.32)\end{array}$ & $\begin{array}{l}-1.069 \\
(.109)\end{array}$ & .056 \\
\hline $\begin{array}{l}\text { Circunferencia } \\
\text { cintura }\end{array}$ & $\begin{array}{l}98.80 \\
(9.90)\end{array}$ & $\begin{array}{l}97.80 \\
(7.12)\end{array}$ & $\begin{array}{l}-.552 \\
(.581)\end{array}$ & .104 & $\begin{array}{c}97.32 \\
(14.69)\end{array}$ & $\begin{array}{c}95.50 \\
(15.77)\end{array}$ & $\begin{array}{l}-1.604 \\
(.109)\end{array}$ & .103 \\
\hline $\begin{array}{l}\text { Circunferencia } \\
\text { cadera }\end{array}$ & $\begin{array}{l}106.00 \\
(5.52)\end{array}$ & $\begin{array}{l}102.80 \\
(4.54)\end{array}$ & $\begin{array}{l}-1.604 \\
(.109)\end{array}$ & .571 & $\begin{array}{l}108.87 \\
(11.87)\end{array}$ & $\begin{array}{l}108.75 \\
(12.20)\end{array}$ & $\begin{array}{l}-.272 \\
(.785)\end{array}$ & .008 \\
\hline
\end{tabular}

Fuente: elaboración propia 
En el GE del pretest al seguimiento se observa un cambio favorable, aunque muy bajo en la HbA1c (ver Tabla 3).

Tabla 3. Comparaciones y tamaño del efecto del CM de la DM2 del GE del pretest al seguimiento

\begin{tabular}{|c|c|c|c|c|}
\hline \multirow[b]{2}{*}{ Variable } & \multicolumn{4}{|c|}{ Grupo experimental $(n=4)$} \\
\hline & $\begin{array}{l}\text { Pretest } \\
M(D E)\end{array}$ & $\begin{array}{l}\text { Seguimiento } \\
\qquad(D E)\end{array}$ & $Z(p)$ & $g$ \\
\hline $\mathrm{HbA1c}$ & $8.43(2.42)$ & $8.02(1.87)$ & $-1.095(.273)$ & .164 \\
\hline Colesterol total & $184.37(15.79)$ & $205.52(22.61)$ & $-1.095(.273)$ & .943 \\
\hline Colesterol HDL & $47.97(13.46)$ & $56.10(10.03)$ & $-1.826(.068)$ & .595 \\
\hline Triglicéridos & $127.47(72.21)$ & $111.20(54.93)$ & $-.730(.465)$ & .220 \\
\hline Peso & $70.95(8.61)$ & $67.70(5.90)$ & $-1.461(.144)$ & .382 \\
\hline Circunferencia cintura & $97.32(14.69)$ & $93.00(14.89)$ & $-1.604(.109)$ & .253 \\
\hline Circunferencia cadera & $108.87(11.87)$ & $105.00(11.22)$ & $-1.841(.066)$ & .291 \\
\hline
\end{tabular}

Fuente: elaboración propia

Con relación a las variables psicológicas asociadas al CM de la DM2 en el GE del pretest al seguimiento, destaca, como se observa en la Tabla 4, un efecto favorable muy grande en el malestar emocional asociado a la DM2 $(g=1.399)$.

Tabla 4. Comparaciones y tamaño del efecto de las variables psicológicas asociadas al CM de la DM2 del GC y del GE del pretest al postest

\begin{tabular}{|c|c|c|c|c|c|c|c|c|}
\hline \multirow[b]{2}{*}{ Variable } & \multicolumn{4}{|c|}{ Grupo control $(\mathrm{n}=5)$} & \multicolumn{4}{|c|}{ Grupo experimental $(n=4)$} \\
\hline & $\begin{array}{c}\text { Pretest } \\
M \\
(D E)\end{array}$ & $\begin{array}{c}\text { Postest } \\
M \\
(D E)\end{array}$ & $\begin{array}{l}\mathrm{Z} \\
(p)\end{array}$ & $g$ & $\begin{array}{c}\text { Pretest } \\
M \\
(D E)\end{array}$ & $\begin{array}{c}\text { Postest } \\
M \\
(D E)\end{array}$ & $\begin{array}{c}Z \\
(p)\end{array}$ & $g$ \\
\hline MEADM & $\begin{array}{l}10.60 \\
(6.42)\end{array}$ & $\begin{array}{l}16.40 \\
(8.29)\end{array}$ & $\begin{array}{l}-1.236 \\
(.216)\end{array}$ & .706 & $\begin{array}{l}51.75 \\
(6.89)\end{array}$ & $\begin{array}{c}24.00 \\
(23.39)\end{array}$ & $\begin{array}{l}-1.826 \\
(.068)\end{array}$ & 1.399 \\
\hline Sintomatología depresiva & $\begin{array}{l}42.60 \\
(4.50)\end{array}$ & $\begin{array}{l}39.60 \\
(4.39)\end{array}$ & $\begin{array}{l}-1.236 \\
(.216)\end{array}$ & .609 & $\begin{array}{c}45.00 \\
(13.68)\end{array}$ & $\begin{array}{c}40.75 \\
(12.60)\end{array}$ & $\begin{array}{l}-1.604 \\
(.109)\end{array}$ & .281 \\
\hline Bienestar psicológico & $\begin{array}{l}181.60 \\
(17.81)\end{array}$ & $\begin{array}{l}171.00 \\
(11.76)\end{array}$ & $\begin{array}{l}-1.355 \\
(.176)\end{array}$ & .634 & $\begin{array}{l}147.25 \\
(11.58)\end{array}$ & $\begin{array}{l}157.75 \\
(25.47)\end{array}$ & $\begin{array}{l}-.365 \\
(.715)\end{array}$ & .461 \\
\hline
\end{tabular}

Fuente: elaboración propia.

En lo que concierne al GE del pretest al seguimiento, se observa un efecto muy grande y favorable en el malestar emocional asociado a la DM2 $(g=1.478)$; con relación al bienestar psicológico, el efecto fue favorable y grande, en tanto que para la sintomatología depresiva fue mediano, también en sentido favorable (ver Tabla 5). 
Tabla 5. Comparaciones y tamaño del efecto del pretest al seguimiento de las variables psicológicas asociadas al CM de la DM2 del GE

\begin{tabular}{|c|c|c|c|c|}
\hline \multirow[b]{2}{*}{ Variable } & \multicolumn{4}{|c|}{ Grupo experimental $(n=4)$} \\
\hline & $\begin{array}{l}\text { Pretest } \\
M(D E)\end{array}$ & $\begin{array}{l}\text { Seguimiento } \\
\qquad(D E)\end{array}$ & $\begin{array}{c}Z \\
(p)\end{array}$ & $g$ \\
\hline MEADM & $51.75(6.89)$ & $20.75(24.85)$ & $\begin{array}{l}-1.826 \\
(.068)\end{array}$ & 1.478 \\
\hline Sintomatología depresiva & $45.00(13.68)$ & $36.00(12.19)$ & $\begin{array}{l}-1.289 \\
(.197)\end{array}$ & .604 \\
\hline Bienestar psicológico & $147.25(11.58)$ & $175(30.82)$ & $\begin{array}{r}-1.461 \\
(.144) \\
\end{array}$ & 1.036 \\
\hline
\end{tabular}

Fuente: elaboración propia.

Respecto la comparación entre grupos, no se identificaron diferencias entre el GE y el GC en el pretest y el postest en los indicadores metabólicos y las variables psicológicas asociadas al CM de la DM2 según la U de Mann-Whitney.

\section{Discusión}

El resultado en este estudio respecto a la $\mathrm{HbAlc}$ coincide con lo encontrado en otras investigaciones realizadas en México, en donde los efectos de las intervenciones cognitivo-conductuales fueron favorables, pero no estadísticamente significativos en este indicador (Hattori, 2014; Montes, Oropeza, Pedroza, Verdugo \& Enríquez, 2013). No obstante, la HbA1c disminuyó, aunque no llegó al rango de control, del pretest al postest y del postest al seguimiento (tres meses), lo cual coincide con estudios como el de Safren et al. (2014) y el de Weinger et al. (2011). Cabe señalar que, en contraste, hay estudios similares que reportan resultados favorables y estadísticamente significativos (Márquez \& Campos, 2001; Ramírez 2013) pero en los cuales se utilizaron muestras más grandes.

El colesterol total fue un indicador que aumentó del pretest al postest y del postest al seguimiento en el GE y fue en esta última medición donde la cifra se ubicó fuera del rango de control (205.52), en tanto que en el GC salió del rango recomendado desde la medición del postest, se requieren intervenciones más precisas para poder controlar este indicador.

Respecto al colesterol HDL en el presente estudio hubo un efecto favorable y progresivo (del pretest al postest y después al seguimiento) al igual que en el trabajo de Weinger et al. (2011). En el caso del GC hubo una disminución de la primera a la segunda medición, lo cual no es favorable clínicamente porque el colesterol HDL tiene un rol protector ante problemas cardiovasculares.

Respecto a los triglicéridos, hubo una mejoría del pretest al postest y a su vez del primero al seguimiento $(<150 \mathrm{mg} / \mathrm{dL})$. Estos resultados son favorables en comparación al GC en donde en ambas mediciones los triglicéridos están en promedio por arriba de lo recomendado. Este indicador es uno de los más complicados de controlar pues está directamente implicado con el consumo de alimentos muy frecuentes en la dieta del mexicano (harinas y azúcares refinados, leche entera, carnes y embutidos ricos en grasa). Por ello, es que en diversos estudios (Del Castillo, 2005; Hattori, 2014; Ramírez 2013) a pesar se obtiene un impacto favorable, no alcanzan diferencias estadísticamente significativas.

Con relación con el peso y su relación directa con el IMC, la perspectiva educativa no funciona para la reducción de peso en pacientes con DM2, el estudio de Barceló, Robles, White, Jadue \& Vega (2001) es muestra de ello, pues la simple transmisión de información no dota de habilidades psicológicas a las personas para que puedan controlar su peso. En contraste, en esta intervención cognitivo-conductual se observó un efecto favorable ene le peso en el GE del pretest al seguimiento con una disminución por arriba de los tres kilogramos, en comparación con el GC, en el cual, aunque también hubo una reducción de peso, esta no llegó al kilogramo. Así, los resultados coinciden con el estudio de Rodríguez, Rentería \& García (2013) en el que también la reducción 
fue de tres kilogramos, y es mayor que el resultado obtenido en el trabajo de Caballero, Gómez \& López (2004).

No se han identificado trabajos en donde se aborden indicadores antropométricos como la circunferencia de cintura y cadera, los cuales son importantes porque se sabe de su relación con las dislipidemias y su consecuente riesgo cardiovascular (Montalbán, 2001).

Respecto a las variables psicológicas (MEADM, sintomatología depresiva y bienestar psicológico) asociadas al CM de la DM2, es necesario señalar que en el diseño experimental de esta investigación se consideraron como variables control. Se observa una disminución estadísticamente significativa y favorable que coincide con trabajos como el Del Castillo (2014), Del Castillo, Guzmán, García \& Martínez, (2012) y Morales (2012).

La sintomatología depresiva disminuyó y se mantuvo este efecto en el periodo de seguimiento a los tres meses en los participantes de la INCOCOM, al igual que otros trabajos reportados (Caballero, Gómez \& López, 2004; Del Castilllo, 2005; Quiroga, 2012; Riveros, Cortazar-Palapa, Alcazar \& Sánchez-Sosa, 2005).

Finalmente, el bienestar psicológico se incrementó paulatinamente del pretest al postest y de este al seguimiento, lo cual coincide con el trabajo de Del Castillo, (2014) y otros trabajos similares en donde se evalúa el impacto en la calidad de vida (Del Castilllo, 2005; Riveros, Cortazar-Palapa, Alcazar \& Sánchez-Sosa, 2005; Robles, 2002).

Esta intervención logró resultados favorables en el colesterol HDL y las variables psicológicas (malestar emocional, sintomatología depresiva y bienestar psicológico), al igual que intervenciones similares; pero con mayor número de sesiones (Pantoja-Magallón, Domínguez-Guedea, Moncada, Reguera, Pérez y Mandujano, 2011; Riveros, Cortazar-Palapa, Alcazar \& Sánchez-Sosa, 2005; Rodríguez, Renteria \& García, 2013; Ybarra, Orozco, De León \& Vargas, 2012). Esto puede ser atribuible, como lo señala Riveros, Cortazar-Palapa, Alcazar \& Sánchez-Sosa (2005) a la naturaleza individual de la intervención, pues las diversas técnicas y actividades se adecuaban a la situación problemática de cada participante.

Es importante abordar el cambio de hábitos alimentarios respecto al tipo de alimentos que se consumen, lo cual está relacionado estrechamente con los lípidos (colesterol total, colesterol HDL y triglicéridos); pues para las personas parece ser más fácil disminuir la cantidad de consumo de alimentos que cambiar el tipo de estos. Por ello, se sugiere que futuras intervenciones prioricen técnicas para dotar de habilidades a los participantes para el cambio del tipo de dieta. Por ejemplo, técnicas que tengan como objetivo hacer más agradables a los alimentos saludables.

\section{Referencias}

Barceló, A., Robles, S., White, F., Jadue, L. \& Vega, J. (2001). Una intervención para mejorar el control de la diabetes en Chile. Revista Panamericana de Salud Pública, 10(5), 328-333.

Caballero, N. P., Gómez, L. G. \& López, T. M. (2004). Diseño, aplicación y evaluación del programa de atención psicológica para adultos mayores con diagnóstico de Diabetes Mellitus tipo 2 o hipertensión (Tesis de Licenciatura). Universidad Nacional Autónoma de México.

Del Castillo, A. (2005). Intervención cognitivo-conductual grupal para mejorar la adherencia terapéutica y la calidad de vida en pacientes con DM2 (Tesis de Maestría). Universidad Nacional Autónoma de México.

Del Castillo, A. (2014). Apoyo social, síntomas depresivos, autoeficacia y bienestar psicológico en pacientes con diabetes tipo 2 (Tesis Doctoral). Universidad Nacional Autónoma de México.

Del Castillo, A., Guzmán, R., García, M. \& Martínez, C. (2012). Intervención cognitivo-conductual para modificar el nivel de distrés en pacientes con diabetes tipo II. En S. Galán, y E. Camacho, Estrés y Salud (pp. 191-208). D. F., México: Manual Moderno.

Del Castillo, A. \& Reyes-Lagunes, I. (2010). Validación convergente y divergente del Cuestionario de Áreas Problema en Diabetes. En García, M., Del Castillo, A., Guzmán, R. M. y Martínez, J. P. (Eds.), Medición en Psicología: del individuo a la interacción (pp. 75-95). Pachuca, Hidalgo: Universidad Autónoma del Estado de Hidalgo.

Federación Internacional de Diabetes. (2015). Atlas de la diabetes de la FID (7a ed.). Recuperado desde http://www.diabetesatlas.org/

Flores, S., Reyes, H., Villalpando, S., Reynoso, N. \& Hernández, M. (2012). Encuesta Nacional de Salud y Nutrición 2012. Evidencia para la política pública en salud. Diabetes en adultos: urgente mejorar la atención y el control. Recuperado desde Internet de Encuesta Nacional de Salud y Nutrición, Instituto Nacional de Salud Pública: http://ensanut.insp.mx/doctos/analiticos/DiabetesMellitus.pdf

García, I. \& Morales, B. V. (2015). Eficacia de la entrevista motivacional para promover la adherencia terapéutica en pacientes con Diabetes Mellitus tipo 2. Universitas Psychologica, 14, 511-522. doi: 10.11144/Javeriana.upsy14-2.eemp 
González-Cantero, J. O. \& Oropeza, R. (2016). Intervenciones cognitivo conductuales para diabéticos en México. Salud Mental, 39, 99-105. doi: 10.17711/SM.0185-3325.2016.006

Hattori, M. (2014). Entrenamiento en competencias de afrontamiento al adulto mayor con diabetes mellitus tipo 2 y su cuidador informal (Tesis Doctoral). Universidad Nacional Autónoma de México.

Lucha-López, M. O., Lucha-López, A. C., Tricás-Moreno, J. M., Salavera-Bordás, C., Estébabez, E. \& Vidal-Peracho, C. (2017). Impacto de un programa cuerpo-mente basado en ejercicios de fisioterapia y técnicas psicológicas en el bienestar psíquico de una población de pacientes diabéticos tipo 2. Universitas Psychologica, 16, 1-13. doi: 10.11144/Javeriana.upsy16-1.ipcb

Márquez, B. E. \& Campos M. V. (2001). Intervención cognitivo-conductual dirigido a pacientes diabéticos tipo II (Tesis de Licenciatura). Universidad Nacional Autónoma de México.

Medina-Calvillo, M. A., Gutiérrez-Hernández, C. Y. \& Padrós-Blázquez, F. (2013). Propiedades psicométricas de la escala de bienestar psicológico de Ryff en población mexicana. Revista Educación Desarrollo, 27, 25-30.

Montalbán, J. (2001). Índice cintura/cadera, obesidad y estimación del riesgo cardiovascular en un centro de salud de Málaga. Medicina de Familia, 2, 208-215.

Montes, R., Oropeza, R., Pedroza, F. J., Verdugo, J. C. \& Enríquez, J. F. (2013). Manejo del estrés para el control metabólico de personas con diabetes mellitus tipo 2. En-claves del Pensamiento, 7, 67-87.

Morales, O. (2012). Malestar emocional, Estrategias de afrontamiento y control metabólico en pacientes con diabetes tipo 2 (Tesis de Maestría). Universidad Autónoma del Estado de Hidalgo.

Ortiz, M. S., Baeza-Rivera, M. J. \& Myers, H. F. (2013). Propiedades psicométricas de la Escala de Estrés para Diabéticos en una muestra de pacientes diabéticos tipo II chilenos. Terapia psicológica, 31, 281-286.

Ortiz, M., Ortiz, E., Gatica, A. \& Gómez, D. (2011). Factores Psicosociales Asociados a la Adherencia al Tratamiento de la Diabetes Mellitus Tipo 2. Terapia psicológica, 29, 5-11.

Pantoja-Magallón, C. E., Domínguez-Guedea, M. T., Moncada, E., Reguera, M. E., Pérez, M. S. \& Mandujano, M. F. (2011). Programa de entrenamiento en habilidades de autocontrol alimenticio en personas diabéticas e hipertensas. Revista Electrónica de Psicología Iztacala, 14, 98-115.

Polonsky, W. H., Anderson, B. J., Lohrer, P. A., Welch, G., Jacobson, A. M., Aponte, J. E. \& Schwartz, C. E. (1995). Assessment of diabetes-related distress. Diabetes Care, 18, 754-760.

Quiroga, A. (2012). Intervención telefónica para promover la adherencia terapéutica en pacientes diabéticos con síntomas de ansiedad y depresión. Enseñanza e Investigación en Psicología, 17, 387-403.

Ramírez, M. (2013). Intervención psicoeducativa cognitivo-conductual en pacientes con diabetes mellitus tipo 2 (Tesis de Licenciatura). Universidad Nacional Autónoma de México.

Rivera, B. M., Corrales, A. E. \& Piña, J. A. (2007). Validación de la Escala de Depresión de Zung en Personas con VIH. Terapia Psicológica, 25, 135-140.

Riveros, A., Cortazar-Palapa, J., Alcazar, F. \& Sánchez-Sosa, J. J. (2005). Efectos de una intervención cognitivo-conductual en la calidad de vida, ansiedad, depresión y condición médica de pacientes diabéticos e hipertensos esenciales. International Journal of Clinical and Health Psychology, 5, 445-462.

Robles, R. (2002). Evaluación y modificación de la calidad de vida de personas con diabetes mellitus tipo II (Tesis doctoral). Recuperada desde http://132.248.9.195/pdtestdf/0312723/Index.html

Rodríguez, M. L., Rentería, A. \& García, J. C. (2013). Adherencia a la dieta en pacientes diabéticos: efectos de una intervención. Summa Psicológica UST, 10, 91-101.

Ryff, C. D. (1989). Happiness is everything, or is it? Explorations on the meaning of psychological well-being. Journal of Personality and Social Psychology, 57, 1069-1081.

Safren, S. A., Gonzalez, J. S., Wexler, D. J., Psaros, C., Delahanty, L. M., Blashill, A. J. ... Cagliero, E. (2014). A Randomized Controlled Trial of Cognitive Behavioral Therapy for Adherence and Depression (CBTAD) in p atients with uncontrolled type 2 diabetes. $\mathrm{Di}$ abetes Care, 37, 625-633. doi: 10.2337/dc13-0816

Sociedad Mexicana de Psicología (2010). Código Ético del Psicólogo. D. F., México: Trillas.

Torres, A. M. \& Piña, J. A. (2010). Asociación entre variables psicológicas y sociales con la adhesión en personas con diabetes tipo 2. Terapia psicológica, 28, 45-53.

Weinger, K., Beverly, E. A., Lee, Y., Sitnokov, L., Ganda, O. P. \& Caballero, E. (2011). The effect of a structured behavioral intervention on poorly controlled diabetes. Archives of Internal Medicine, 171, 1990-1999. doi: 10.1001/archinternmed.2011.502

Ybarra, J. L., Orozco, L. A., De León, A. E. \& Vargas, A. C. (2012). Intervención Cognitivo-Conductual para la mejora del Autocuidado y la Calidad de Vida en adolescentes con Diabetes Tipo 1 y sus familias. Revisa Latinoamericana de Medicina Conductual, 2, 96-102.

Zung, W. W. K. (1965). A self-rating depression scale. Archives of General Psychiatry, 12(63), 63-70. 